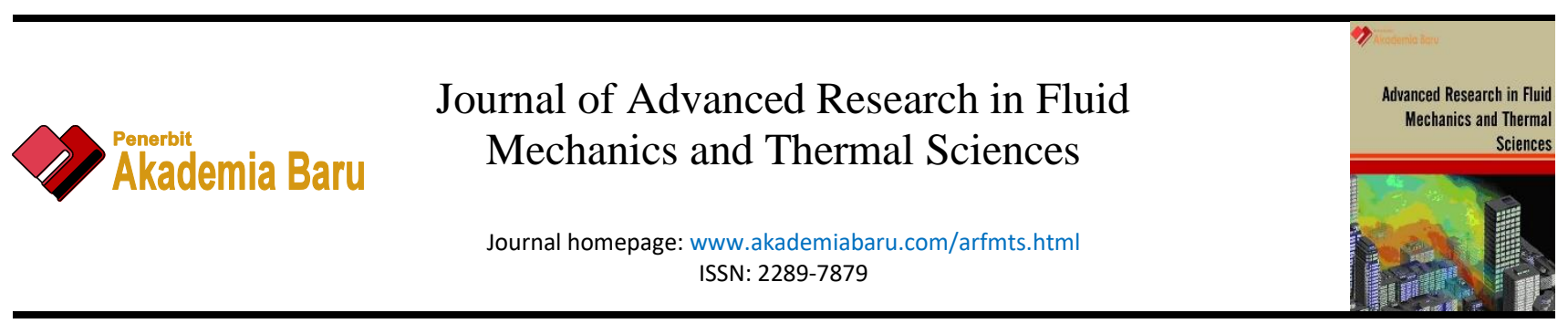

\title{
A Comparative Study for Different Shapes of Airfoils
}

\author{
Mohamed Adel El Hady ${ }^{1, *}$ \\ 1 Department of Mechanical Power Engineering, Faculty of Engineering, Zagazig University, Zagazig, 44519, Egypt
}

\section{ARTICLE INFO ABSTRACT}

\section{Article history:}

Received 31 July 2019

Received in revised form 30 November 2019

Accepted 2 December 2019

Available online 7 April 2020

\section{Keywords:}

NACA airfoil; lift coefficient; drag

coefficient; CFD; simulation; pressure distribution

\begin{abstract}
Lift and drag forces on an airfoil blade or wing are known to depend mainly on the airfoil geometry in addition to the angle of attack. In this paper a comparative study of lift and drag characteristics and pressure distribution for airfoils of different geometries was carried out. Three different geometries, NACA0012, NACA2412, and SG6043 were investigated. A 2-D numerical analysis was performed using ANSYS 16. The $k-\omega$ shear stress transport (SST) model was used to simulate the effect of turbulence. The numerical simulation performed on the flow field around the three geometries considered here was applied at different angles of attack: $-10,-5,0,5,10,15,20$, and 25. Pressure distributions, and lift and drag coefficients were obtained for each angle. The numerical results obtained were validated via comparison of the predicated data with published results in the literature. From this comparison, showing good agreement, the use of the SG6043 airfoil in wind turbine applications is recommended, in view of its high (lift/drag) ratio. the results showed also the optimum values for lift to drag ratio for the different three profiles.
\end{abstract}

Copyright @ 2020 PENERBIT AKADEMIA BARU - All rights reserved

\section{Introduction}

The aerodynamic characteristics of an airfoil-section blade is known to be highly affected by the airfoil geometry and the operating conditions. The airfoil-constructed blade geometry includes: the type of base profile used, the camber angle, the blade span/chord ratio (aspect ratio), and the twist angle. On the other hand, the operating conditions include: the flow Mach number, the ambient conditions (pressure, temperature, and turbulence level), and angle of attack (AoA). Lift and drag forces are generated with values depending on airfoil blade geometry and operating conditions. The lift force, perpendicular to the chord line is a component of the resultant force caused by the pressure difference between the lower and upper surface of the airfoil blade. The other component which is parallel to the chord line is called the pressure-induced drag force. This drag component is to be

\footnotetext{
* Corresponding author.

E-mail address: engm_adel@yahoo.com (Mohamed Adel El Hady)
} 
added to the friction-induced drag component. In aircraft applications, it is sought to increase the gross lift force (by all lift-generating surfaces) and to decrease the gross drag force (by all draggenerating surfaces). This means raising the lift/drag ratio. In other words, the aerodynamic performance of an aircraft depends largely on the lift and drag characteristics of the aircraft and its wing geometries, when operating under certain conditions.

\section{Previous Works}

Different researches have been conducted on the flow field around an airfoil with different geometries and various operating conditions. These researches have investigated the flow field experimentally as well as numerically. For example, Hossain et al., [1] have investigated experimentally the aerodynamic characteristics of a rectangular wing with and without bird feather as winglets for several flow velocities. Their results show that the drag coefficient decreases by $25-$ $30 \%$ and the lift coefficient increases by $10-20 \%$ when utilizing bird feather at 8-degree angle of attack. Mineck et al., [2] have examined three planar, untwisted wings with the same elliptical chord but and different curvatures of the quarter-chord line. They show that the elliptical wing with unswept quarter-chord line has the lowest lifting performance, whereas the elliptical wing with the unswept trailing edge has the highest lifting performance. Moreover, the crescent-shaped wing has moderate performance in between. Haque et al., [3] have analyzed experimentally the performance of NACA 4412 airfoil. They tested two models, one with a straight leading and trailing edges, i.e. having a rectangular planform and the other model with a straight trailing edge and a curved leading edge. The results they obtained show that the model with a curved leading-edge planform has higher lift to drag ratio than the other model. Mahmud [4] has analyzed the effectiveness of an airfoil with bi-camber surface. Kandwal et al., [5] has studied the fluid flow and aerodynamic forces over a twodimensional NACA 4412 airfoil. Robert [6] has studied the variation of pressure distribution over NACA 4412 airfoil with different Reynolds number. Sharma [7] has analyzed the flow behavior around an airfoil-shaped body. The aerodynamic characteristics $\left(C_{L}, C_{D}\right.$ and $\left.L / D\right)$ can be calculated from the surface pressure distribution of the wing, as shown by Anderson [8]. Venkatesan et al., [9] have been able to enhance the lift and drag for NACA 2412 airfoil by introducing dimples. They simulated dimples of various geometrical shapes (square, rectangle and triangle) using ANSYS CFX. They show that dimples with square shape give the best results. Sogukpinar $[10,11]$ have studied effects of NACA 0012 airfoil modification on aerodynamic performance. In their numerical analysis, they varied the thickness of NACA 0012 from lower surface and different relative thicknesses for modified asymmetrical airfoils. Sogukpinar [12] has investigated the aerodynamic performance of S-series wind turbine airfoil of S 825 to find the optimum angle of attack. He used RANS and SST turbulence models in his CFD analysis. The comparison indicated that SST turbulence model used in this study could predict aerodynamic properties of wind blade. Yemenici [13] has made an experimental investigation of the flow field around NACA0012 airfoil, studying the effects of varying the Reynolds number and the angle of attack. His results show that the pressure and lift coefficients depend strongly on Reynolds number and attack angle. Rajakumar [14] has examined numerically the lift and drag forces in a wind turbine blade (NACA 4420 airfoil) at different sections for the effect of angle of attack. The analysis shows that at an angle of attack of 50 the Lift/Drag ratio is highest. Mehdi [15] has investigated the influence of Reynolds number and angle on the aerodynamic performance of two dimensional NACA 4412 airfoil. He considered different Reynolds numbers and angles of attack, showing that the highest lift coefficient is obtained at $R e=4 \times 10^{6}$ for an angle of attack of $9^{\circ}$, whereas the lowest drag coefficient occurs at $\mathrm{Re}=1 \times 10^{6}$ and an angle of attack of $0^{\circ}$. It's clear that all previous researches have examined different profiles for the airfoil but in a separate comparison. No 
recommendations have been conducted to use a definite profile due to its advantage over the others. So, the main objective of this paper is to simulate the flow field around a 2-D airfoil with different geometries, namely: NACA0012, NACA2412, and SG6043. Different angles of attack were considered for each profile. CFD results were validated by comparing the present results with previous works. Pressure, temperature, and velocity distributions are presented here. Finally, comparisons between the different profiles examined are presented and discussed.

\section{Theoretical Background}

The governing equations of the flow system considered are the basic equations of fluid dynamics (conservation of mass, conservation of momentum, and conservation of energy). In addition to these basic equations, there are some other auxiliary equations (the turbulence model adopted in the numerical solution). The set of five coupled partial differential equations (PDEs) for the conservation of mass, momentum and energy in fluid flows is known as the Navier-Stokes equations. These equations can be presented in both differential and integral forms. Some terms of the full NavierStokes equations may be simplified or ignored if certain assumptions are posed, El-Sayed [16].

\subsection{Continuity Equation}

The mass conservation equation for steady flow is given by

$\frac{\partial}{\partial x_{i}}\left(\rho V_{i}\right)=0$

where $V_{i}$ : The absolute velocity in the $i^{\text {th }}$ direction, $x_{i}$ : The coordinate in the $i^{\text {th }}$ direction, $\rho$ : Fluid density, and $i$ : A tensor indicating 1, 2, 3.

\subsection{Momentum Conservation Equations}

The conservation of momentum equation in the $i^{\text {th }}$ direction for steady flow can be written as

$\frac{\partial}{\partial x_{j}}\left(\rho V_{i} V_{j}\right)=-\frac{\partial p}{\partial x_{i}}+\frac{\partial \tau_{i j}}{\partial x_{j}}$

where $p$ is the static pressure, and $\tau_{i j}$ is the viscous stress tensor given by

$\tau_{i j}=\mu\left[\frac{\partial V_{i}}{\partial x_{j}}+\frac{\partial V_{j}}{\partial x_{i}}-\frac{2}{3} \frac{\partial V_{l}}{\partial x_{l}}\right]$

where $\mu$ : The absolute viscosity and $i, j, l$ : tensor indices indicating $1,2,3$

\subsection{Energy Conservation Equation}

The steady-state equation of conservation of energy is given by

$$
\frac{\partial}{\partial x_{i}}\left[V_{i}(\rho E+p)\right]=\frac{\partial}{\partial x_{i}}\left(K \frac{\partial T}{\partial x_{i}}-\sum_{j} h_{j} J_{i}+\left(V_{i} \tau_{i j}\right)\right)_{h}
$$


where; $E$ : The total energy of the water, $K$ : The fluid thermal conductivity, and $J_{i}$ : The diffusion flux of $j^{\text {th }}$ species in the $i^{\text {th }}$ direction.

The first three terms on the right-hand side of equation (4) represent the energy transfer due to conduction, species diffusion, and thermal energy created by viscous shear in the flow, respectively. The air total energy $E$ is given by

$E=h-\frac{p}{\rho}+\frac{V_{i}^{2}}{2}$

and $h_{j}$ is the specific enthalpy, given by

$h_{j}=\int_{T_{r e f}}^{T} c_{p, j} d T$

where $T_{\text {ref }}=283.24 \mathrm{~K}$

\section{Computational Analysis}

The numerical simulation was performed using ANSYS Fluent 16. The airfoil with different profiles was generated using the Design Modeler. The mesh element density was increased in regions where high computational accuracy was needed, such as the near -wall region of the airfoil. The numerical simulation used the standard K- $\omega$ shear stress transport (SST) model to account for the turbulence effect, Menter [17]. In order to validate the present simulation, a comparison held with NACA0012 results documented by Harris [18] will be presented. The operating conditions considered in the present simulation were: Reynolds number $(\mathrm{Re})=3.0 \times 10^{6}$, free -stream temperature $=283.24 \mathrm{~K}$, density of the air $(\rho)=1.225 \mathrm{~kg} / \mathrm{m}^{3}$, viscosity $(\mu)=1.7894 \times 10^{-5} \mathrm{~kg} / \mathrm{m} . \mathrm{s}$. and angle of attack (AoA) $\alpha=1.86^{\circ}$. Having completed the validation process, various angles of attack are investigated for the three different airfoil profiles: NACA0012, NACA2412, and SG6043.

\section{Boundary Conditions}

In order to simulate the flow around the 2-D airfoil, the domain height and length were set to approximately 15 chord lengths. The mesh size was concentrated around the airfoil to obtain accurate solution as shown in Figure 1. The structured grid consisted of about 95,000 nodes. The influence of mesh size was examined as seen in Figure 2. No variation in the lift coefficient value was observed with further increase in the mesh size beyond 95,000 cells. The structured grid geometry of the airfoil was constructed for the three different airfoils investigated: NACA0012, NACA2412, and SG6043.
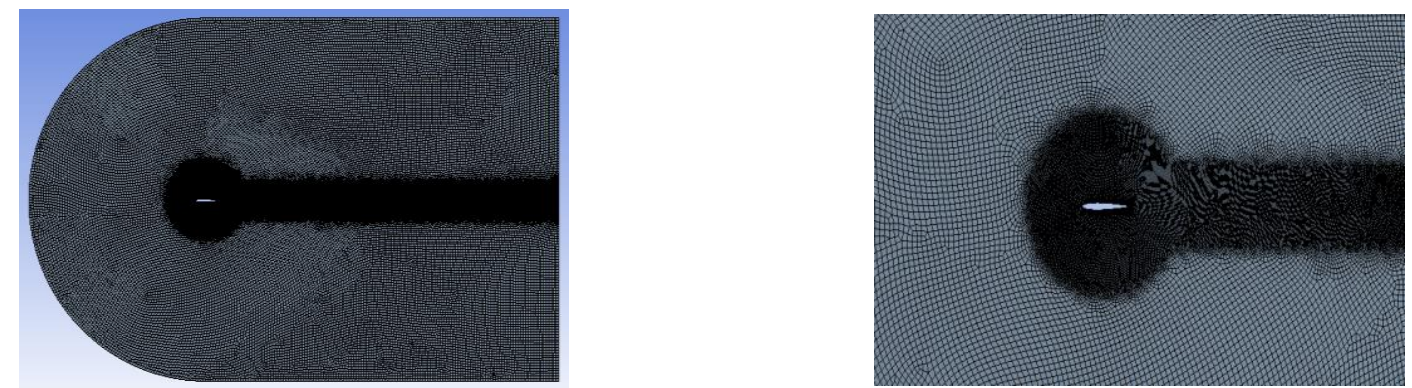

Fig. 1. The mesh geometry generated for NACA0012 airfoil 


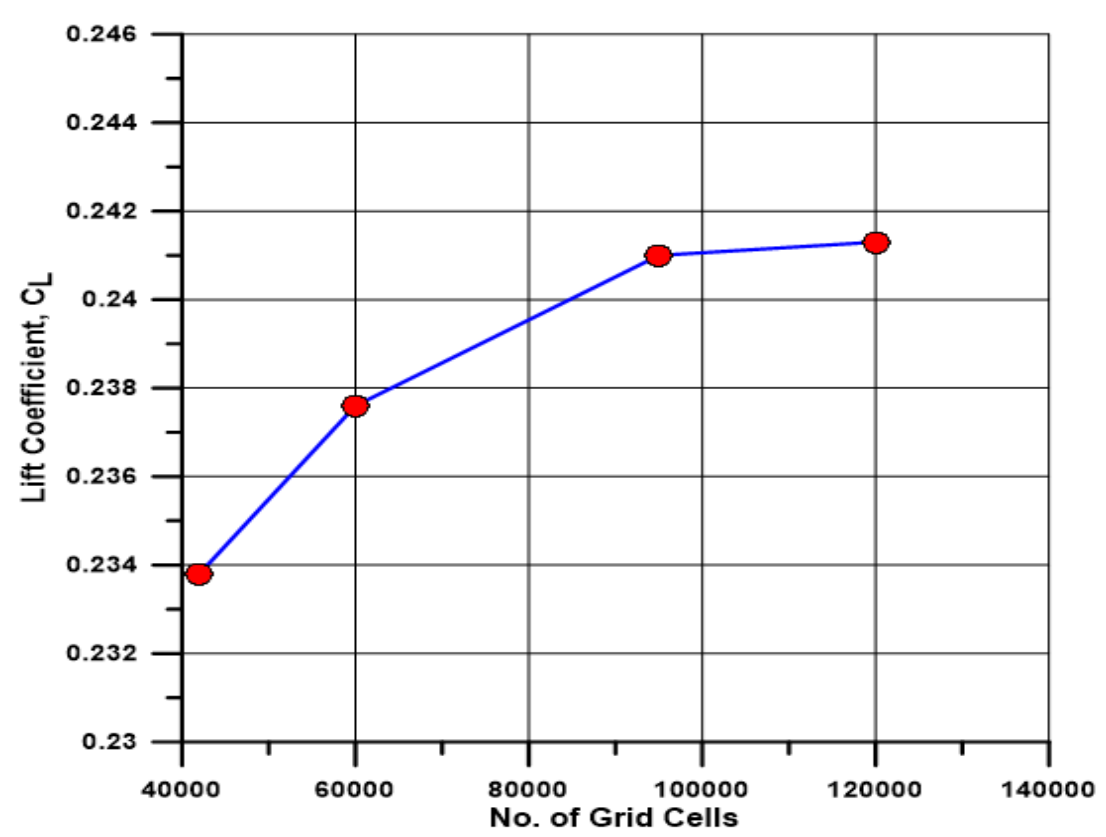

Fig. 2. Variation of lift coefficient with number of grid cells

\section{Validation of Results}

To validate the computation, the numerical results for the 2-D NACA0012 airfoil at AoA $=1.86^{\circ}$ and $5.86^{\circ}$ were compared with the experimental data by Harris [18]. The pressure coefficient distribution around the airfoil by the present study and the results given by Harris is shown in Figure 3 for AoA $=1.85^{\circ}$ and $5.86^{\circ}$ respectively, showing acceptable agreement.

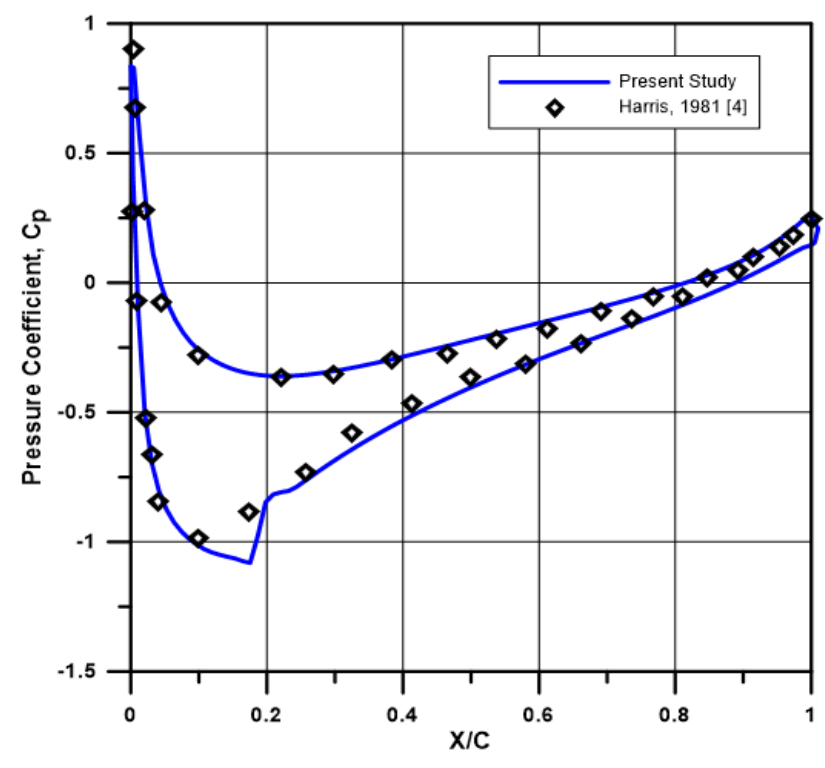

(a)

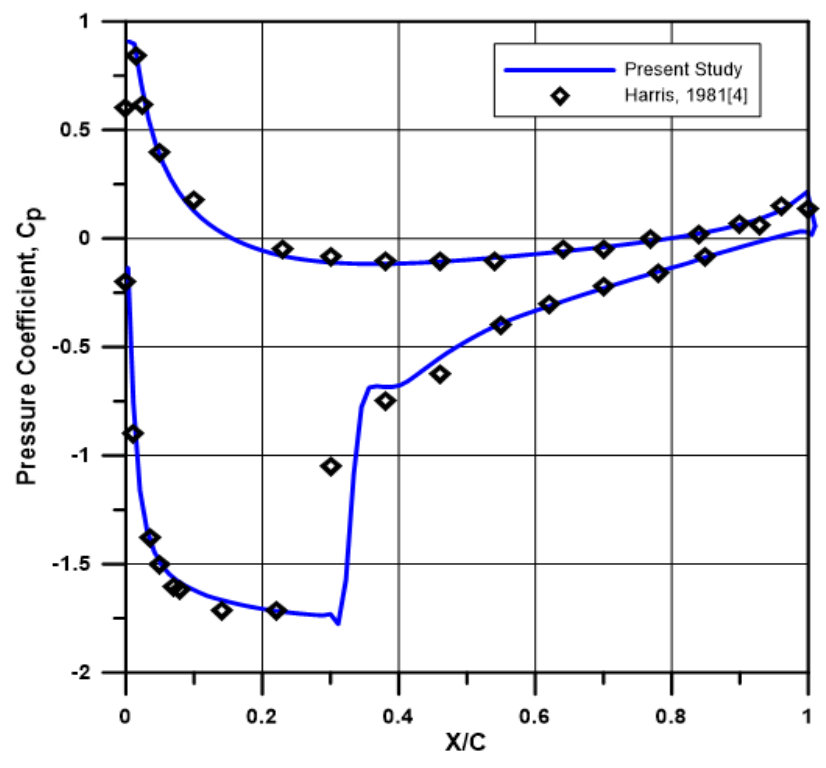

(b)

Fig. 3. Numerical results validation versus previous experimental work (a) Pressure coefficient $\left(C_{p}\right)$ distribution for NACA0012 Airfoil for $A \circ A=1.86^{\circ}$ (b) Pressure coefficient $\left(C_{p}\right)$ distribution for NACA0012 Airfoil for $A o A=5.86^{\circ}$ 


\section{Results and Discussion}

With the computational results validated, different angles of attack ( $A \circ A=-10,-5,0,5,10,15,20$, and $25^{\circ}$ ) were considered and the corresponding results presented. Lift coefficient $\left(C_{L}\right)$ versus angle of attack (AoA) for the three different airfoil profiles are presented in Figure 4. It is observed that the lift coefficient increases with $A \circ A$ up to $A o A=15^{\circ}$. A further increase in AoA leads to decrease in lift coefficient. This is due to the occurrence of flow separation and propagation towards the leading edge as AoA is increased. Consequently, this weak region of separation grows with AoA and the pressure ceases to increase and may even decrease as of the onset of separation onward. Also, it is noticed that SG6043 airfoil has the higher values of lift coefficient over the range of AoA. The effect of angle of attack (AoA) on the drag coefficient is shown in Figure 5 for various airfoils. As the angle of attack increases, the drag coefficient decreases up to $A \circ A=0^{\circ}$, whence a further increase in $A \circ A$ leads to increase in drag coefficient. This is due to the increase in pressure values over the lower surface of the airfoil. The lift to drag ratio is illustrated in Figure 6., where the lift to drag ratio appears to increase with AoA up to $A O A=5^{\circ}$ for $S G 6043$ and up to $A O A=10^{\circ}$ for both NACA0012 and NACA2412. A further increase in AoA beyond these values causes decrease in lift to drag ratio. It is observed that among the three profiles, the SG6043 profile has the highest value of lift to drag ratio. For this reason, this latter profile is preferred for wind turbine applications.

The pressure coefficient $\left(C_{p}\right)$ distributions on NACA0012, NACA2412, and SG6043 airfoils for various angles of attack ( $A \circ A)$ are shown in Figure 7, 8 and 9, respectively.

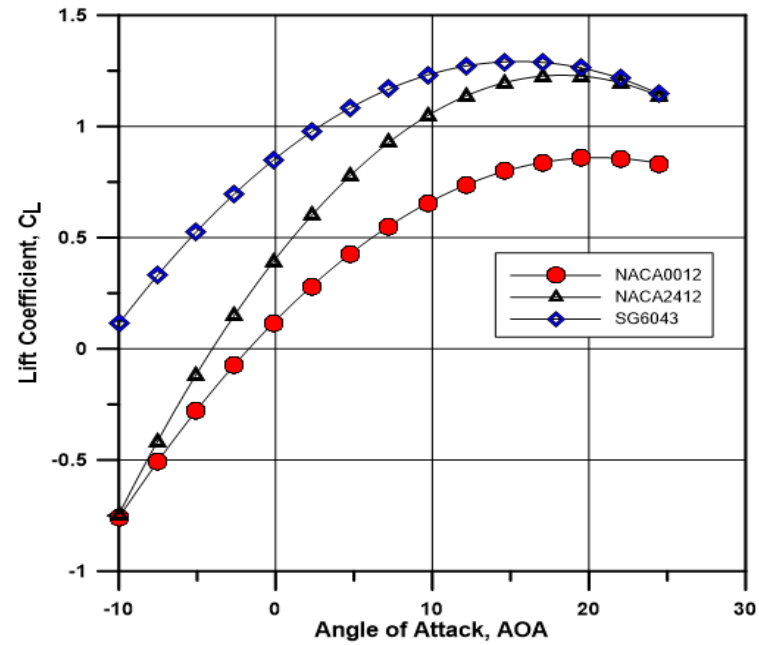

Fig. 4. Lift coefficient $\left(C_{L}\right)$ versus angle of attack (AoA)

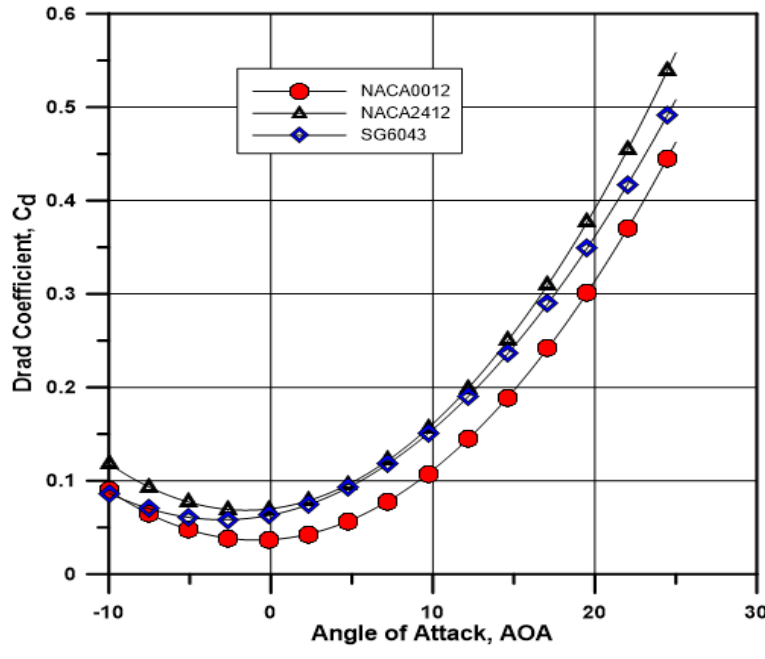

Fig. 5. Drag coefficient $\left(C_{D}\right)$ versus angle of attack (AoA)

Negative pressure prevails on the upper surface of the airfoil whereas positive pressure appears on the lower surface, thus generating the lift force. The pressure values on the upper surface of the airfoil decrease with AoA, resulting from the early flow separation on the airfoil surface as the angle of attack increases. As the flow separation point occurs early, by effect of the adverse pressure gradient, a weak flow region starts and grows with AoA. Also, it can be concluded that the AoA of $20^{\circ}$ gives the lowest pressure values over the upper surface, giving the highest lift coefficient seen in Figure 4. 


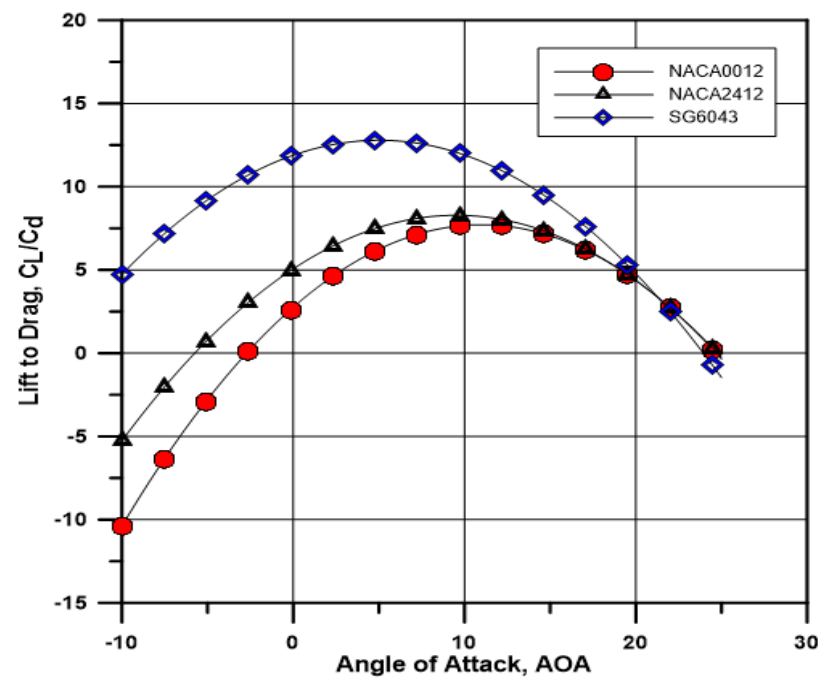

Fig. 6. Lift to Drag $\left(C_{L} / C_{D}\right)$ versus angle of attack (AoA)

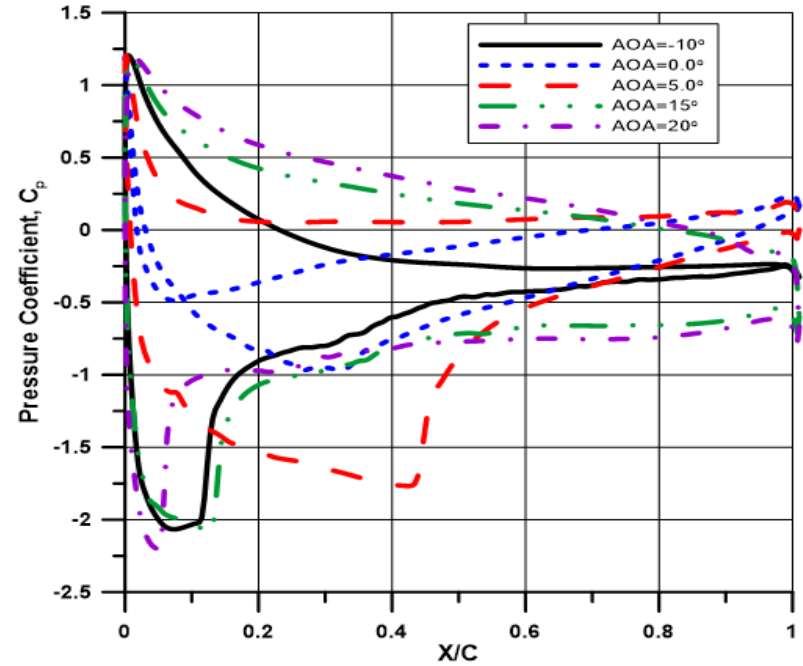

Fig. 8. Pressure coefficient $\left(C_{p}\right)$ distribution on NACA2412 airfoil for different angles of attack (AoA)

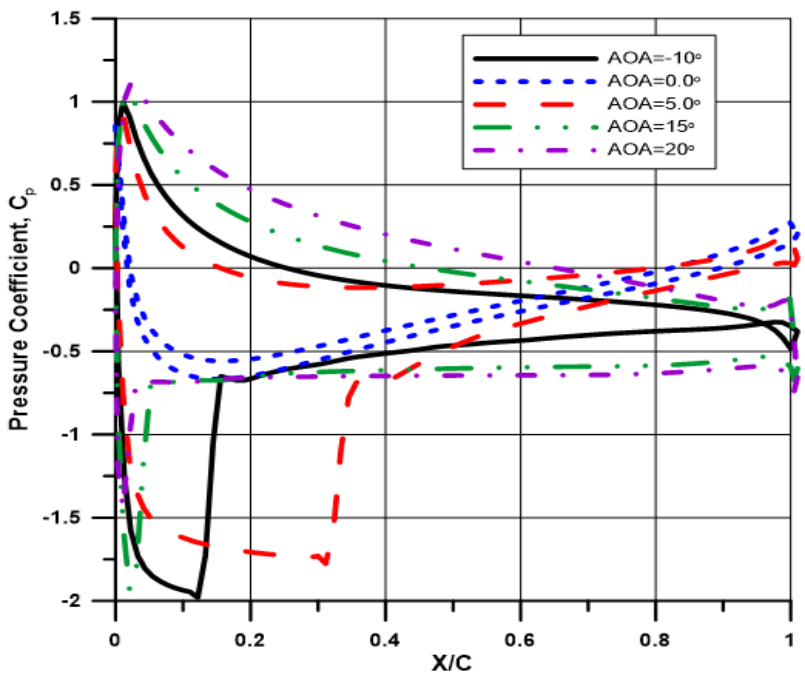

Fig. 7. Pressure coefficient $\left(C_{p}\right)$ distribution on NACA0012 airfoil for various angles of attack (AoA)

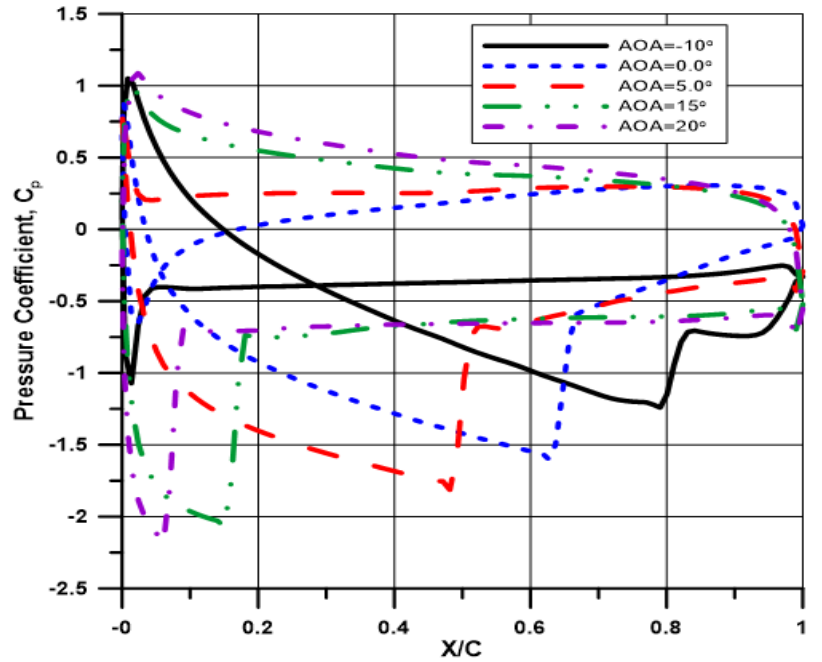

Fig. 9. Pressure coefficient $\left(C_{p}\right)$ distribution on SG6043 Airfoil for different angles of attack (AoA)

Turbulent kinetic energy and velocity contours on NACA0012, NACA2412, and SG6043 airfoils are shown in Figure 10 through Figure 15. It is observed that, for $A \circ A=-10^{\circ}$, low turbulent kinetic energy and velocity region occur on the lower surface. This is due to the occurrence of flow separation on the lower surface due to the high decrease in AoA. Also, it is noticed as the angle of attack increases the low region of turbulent kinetic energy shifts from the lower surface to the upper surface due to the corresponding shift of the separation point from lower surface to upper surface. So, the AoA of $20^{\circ}$ gives the largest turbulent kinetic energy region due to the low velocities occurring in this weak area. For SG6043 airfoil, the turbulent kinetic energy and velocity are generated intensively compared with NACA0012 and NACA2412 for the different angles of attack. Also, the turbulent kinetic energy and velocity contours are asymmetric around the airfoil in contrast with the other profiles. This is due to the asymmetry of the airfoil itself, thus interpreting the high values of lift coefficient over the range of the angle of attack (AoA) in this case. 


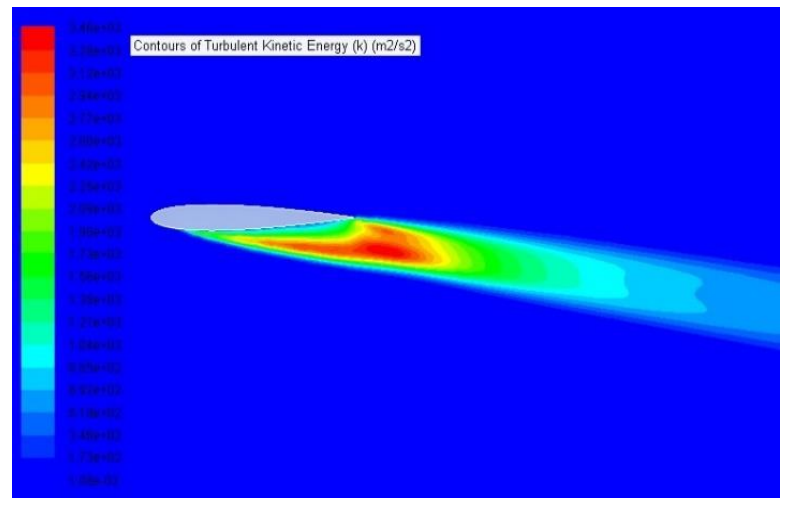

(a)

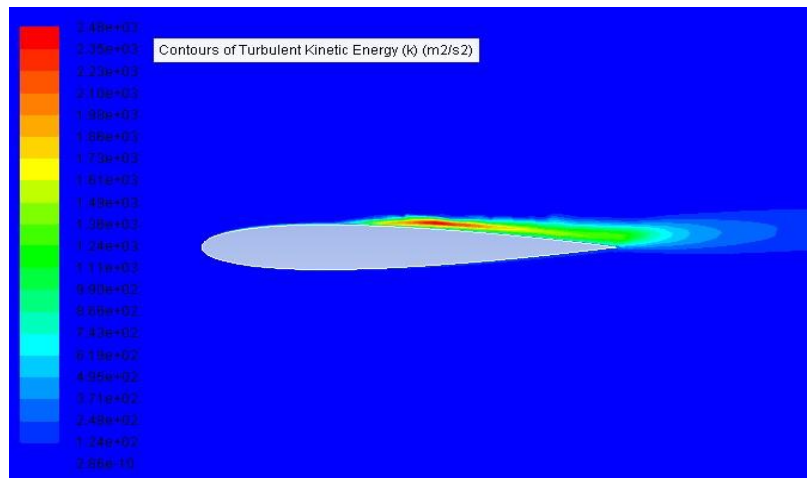

(c)

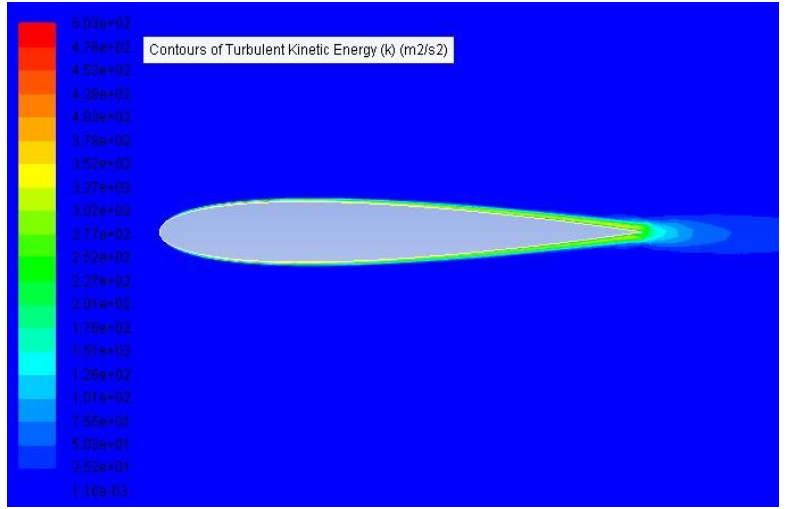

(b)

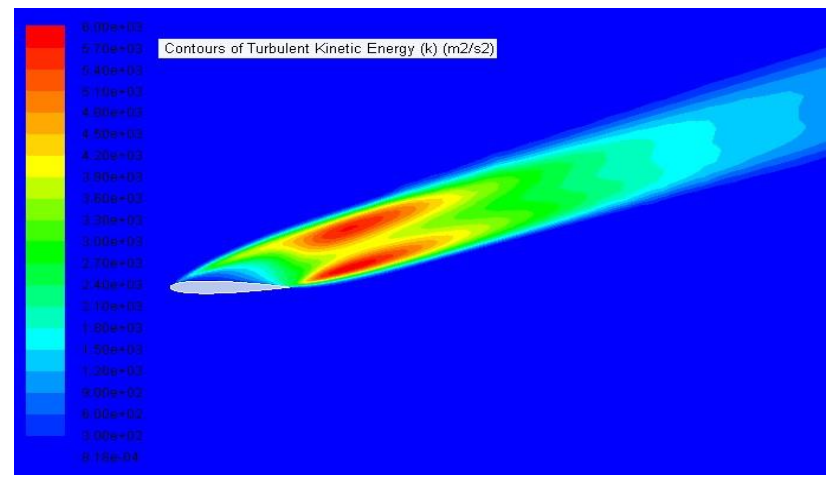

(d)

Fig. 10. Turbulent Kinetic Energy contours around the NACA0012 Airfoil (a) $\alpha=-10^{\circ}$ (b) $\alpha=0^{\circ}$ (c) $\alpha=5^{\circ}$ (d) $\alpha=20^{\circ}$

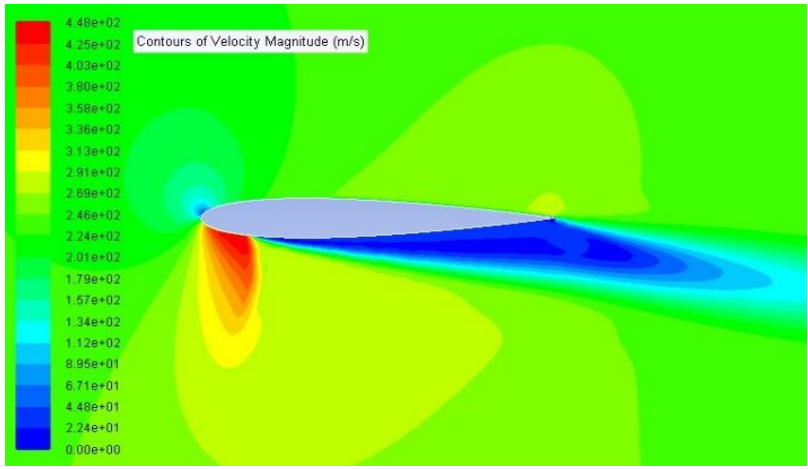

(a)

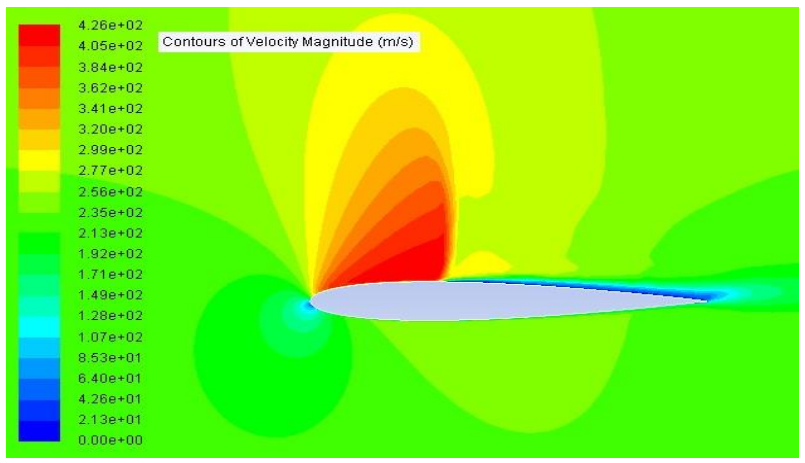

(c)

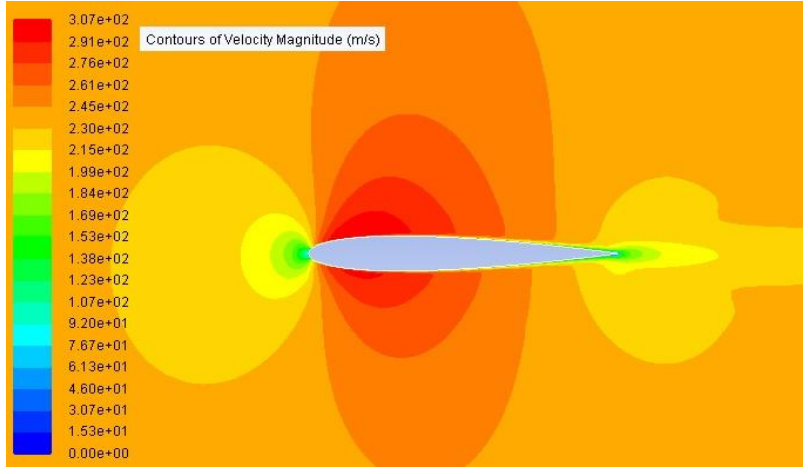

(b)

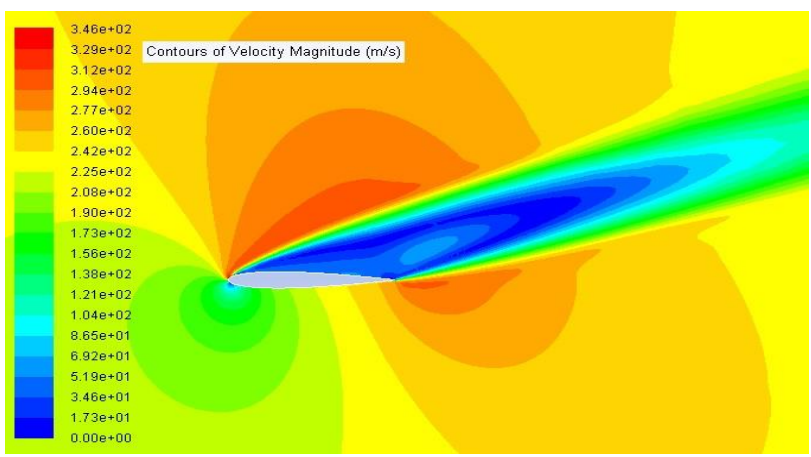

(d)

Fig. 11. Velocity contours around the NACA0012 Airfoil (a) $\alpha=-10^{\circ}$ (b) $\alpha=0^{\circ}$ (c) $\alpha=5^{\circ}$ (d) $\alpha=20^{\circ}$ 


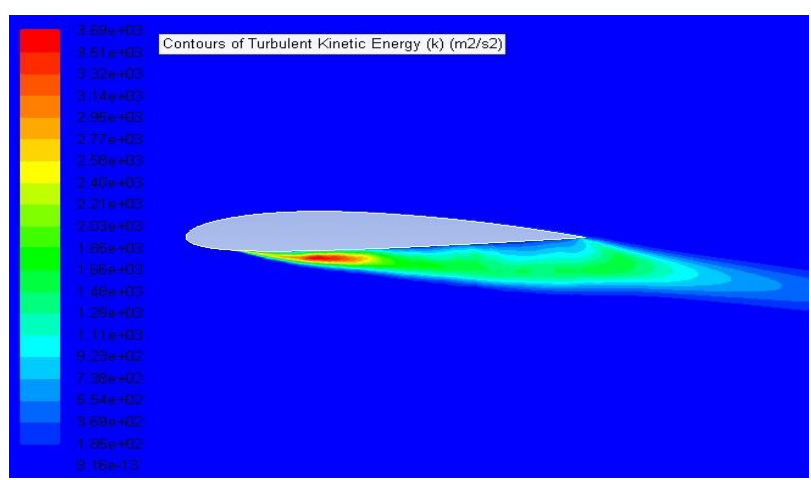

(a)

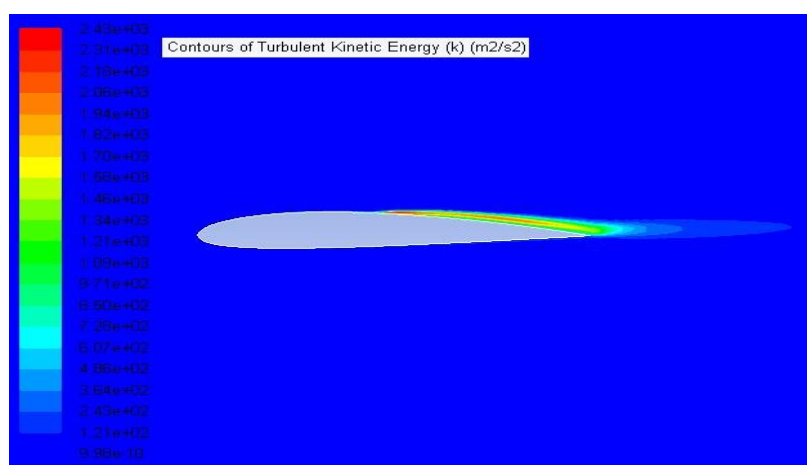

(c)

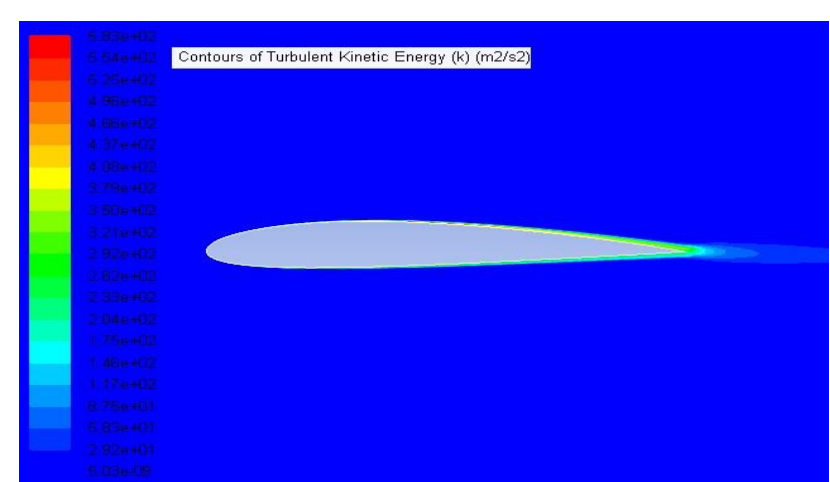

(b)

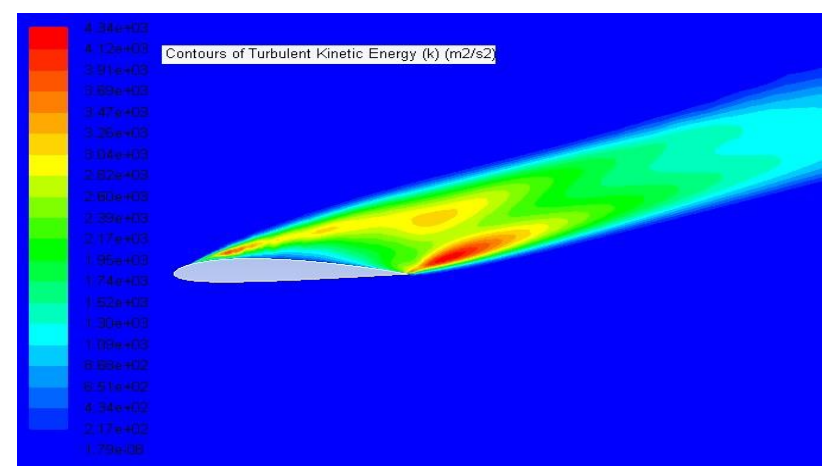

(d)

Fig. 12. Turbulent Kinetic Energy contours around the NACA2412 Airfoil (a) $\alpha=-10^{\circ}$ (b) $\alpha=0^{\circ}$ (c) $\alpha=5^{\circ}$ (d) $\alpha=20^{\circ}$

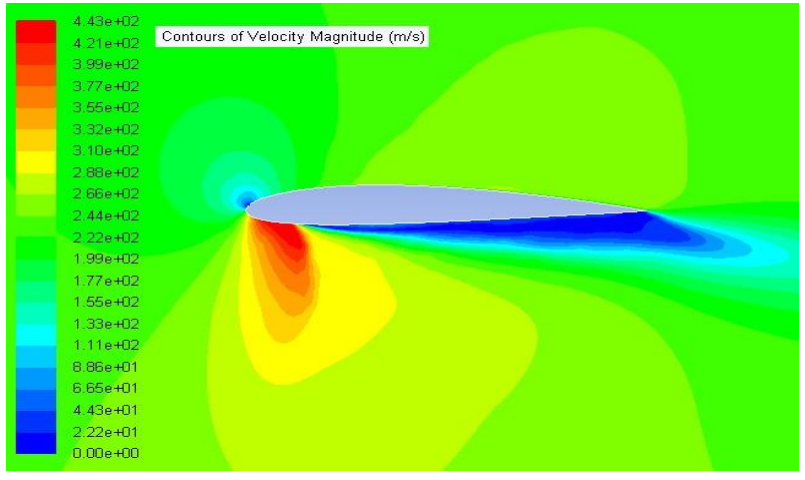

(a)

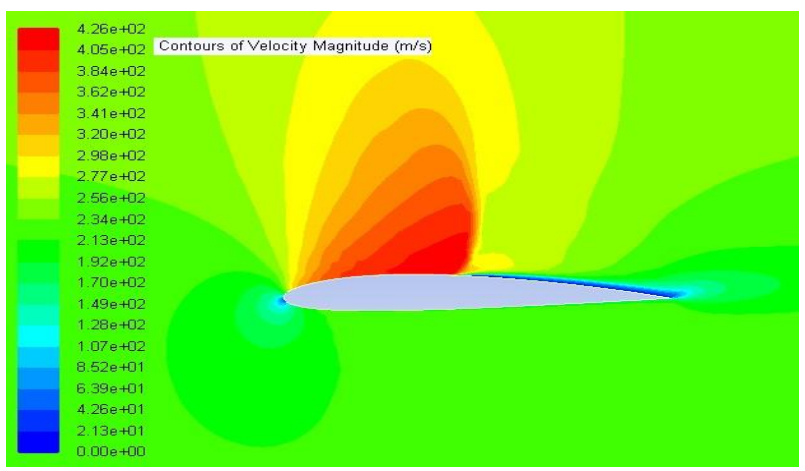

(c)

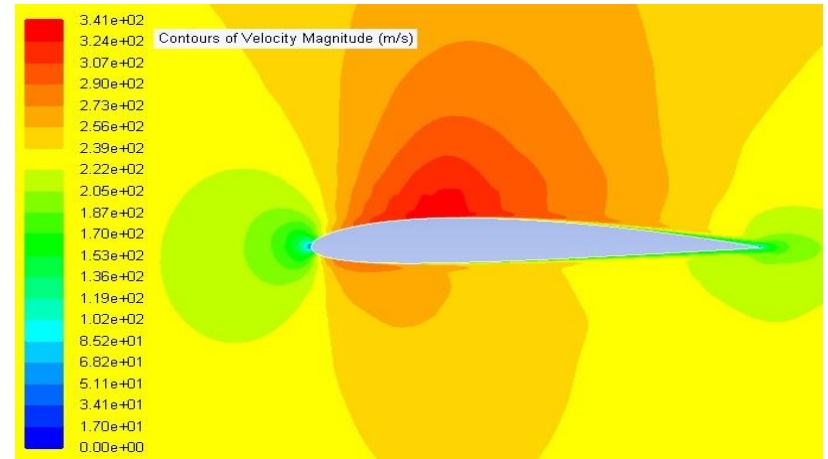

(b)

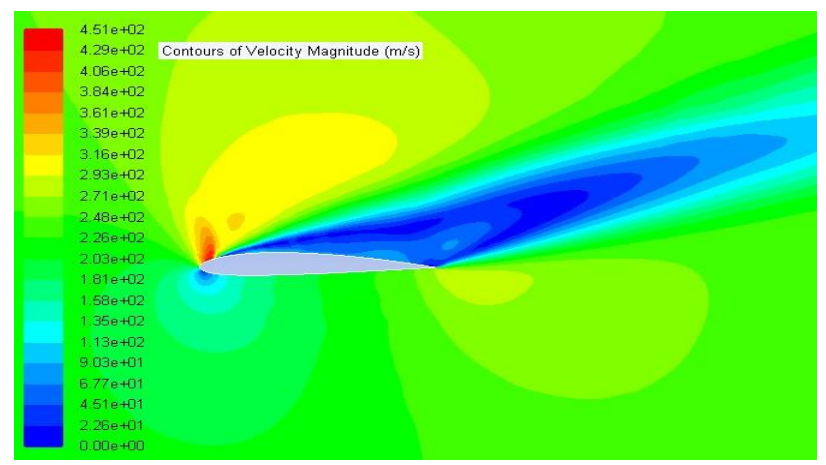

(d)

Fig. 13. Velocity contours around the NACA2412 Airfoil (a) $\alpha=-10^{\circ}$ (b) $\alpha=0^{\circ}$ (c) $\alpha=5^{\circ}$ (d) $\alpha=20^{\circ}$ 


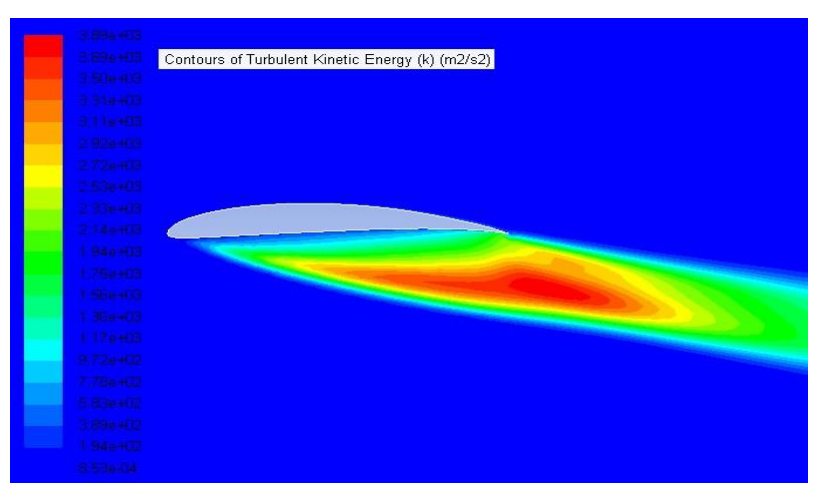

(a)

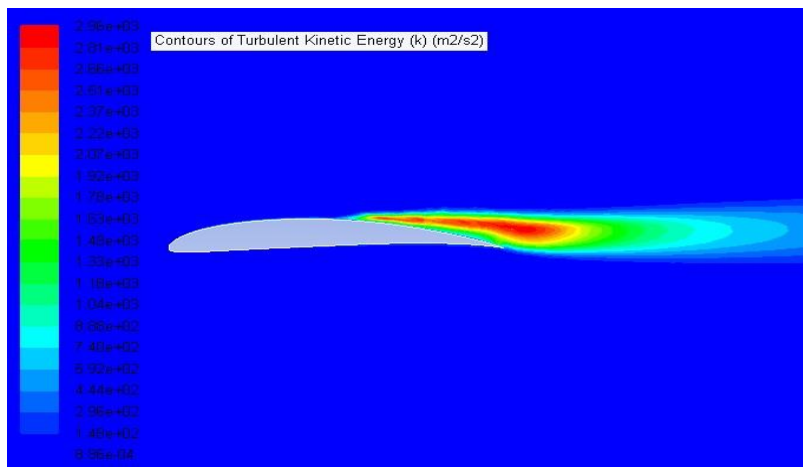

(c)

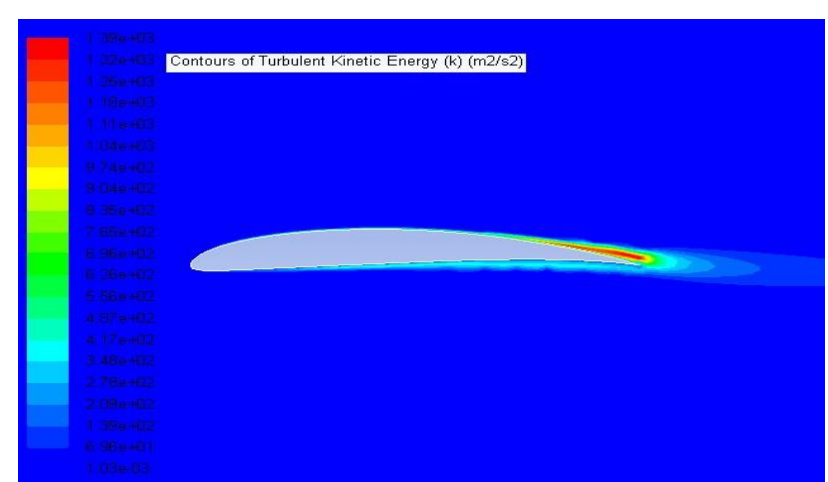

(b)

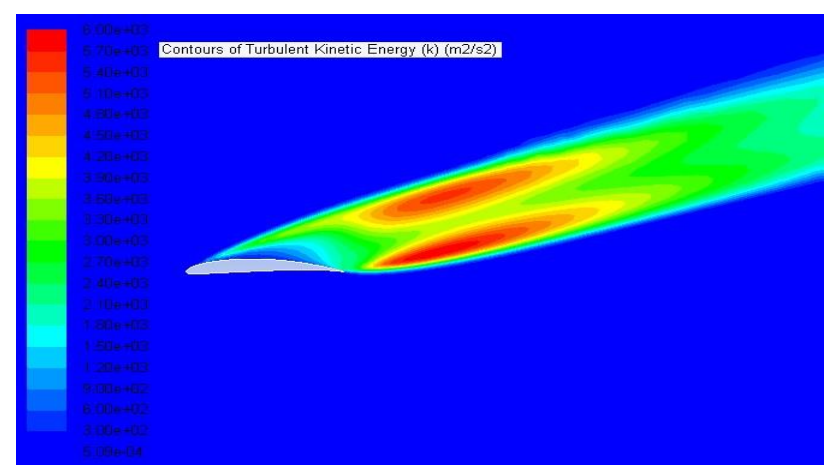

(d)

Fig. 14. Turbulent Kinetic Energy contours around the SG6043 Airfoil (a) $\alpha=-10^{\circ}$ (b) $\alpha=0^{\circ}$ (c) $\alpha=5^{\circ}$ (d) $\alpha=20^{\circ}$

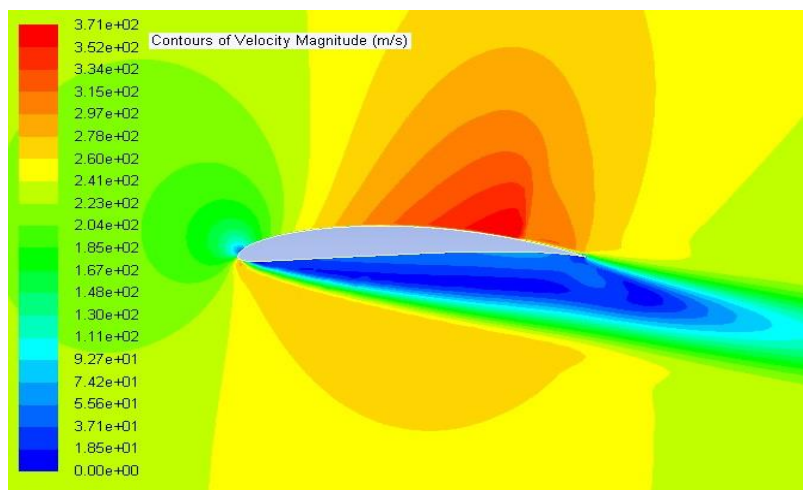

(a)

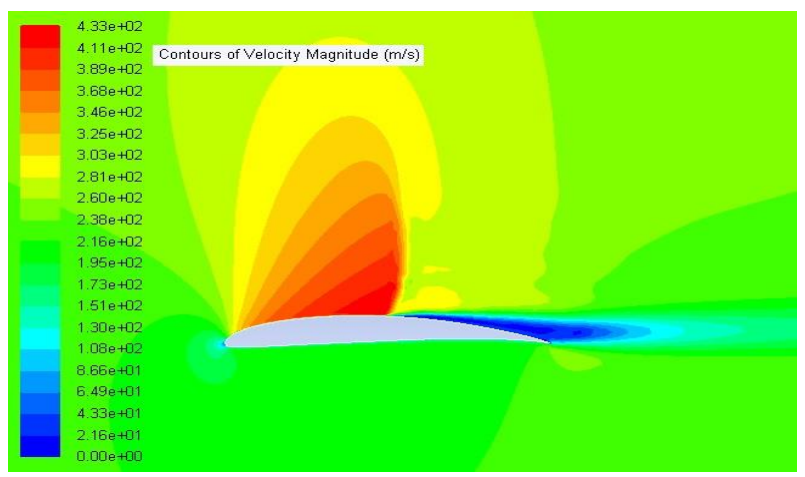

(c)

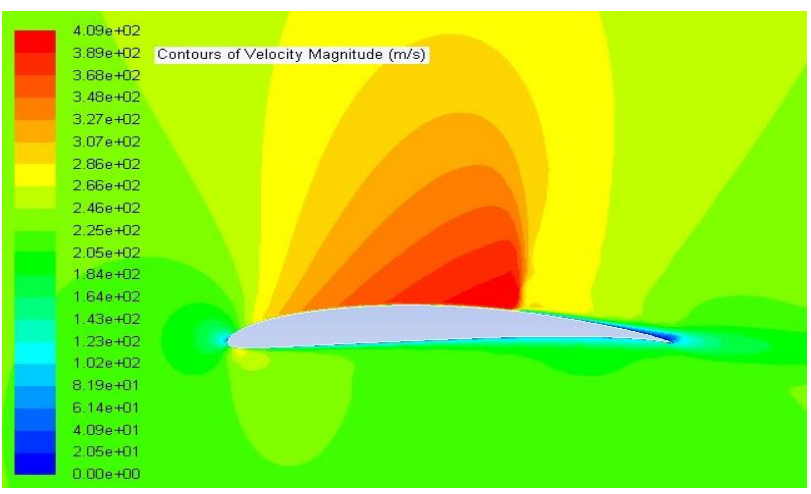

(b)

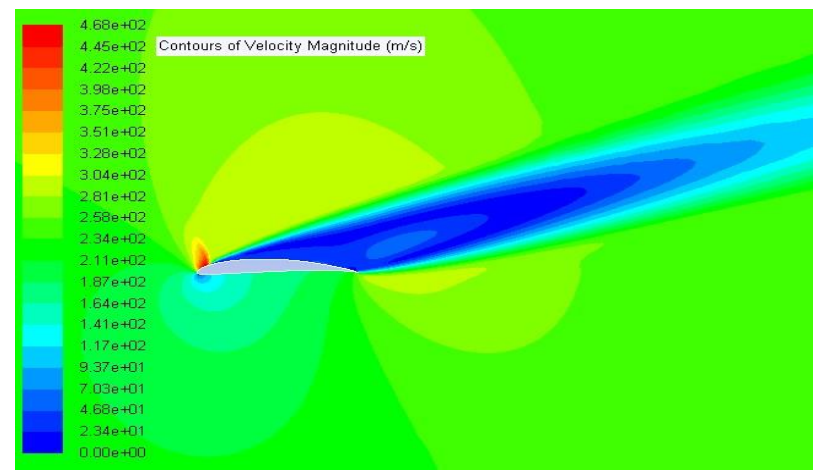

(d)

Fig. 15. Velocity contours around the SG6043 Airfoil (a) $\alpha=-10^{\circ}$ (b) $\alpha=0^{\circ}$ (c) $\alpha=5^{\circ}$ (d) $\alpha=20^{\circ}$ 


\section{Summary and Conclusions}

A numerical analysis was performed to simulate the flow around three different shapes of airfoils NACA0012, NACA2412, and SG6043. The numerical simulation was conducted using ANSYS Fluent 16.0 which solves the Navier-Stokes and energy equations to predict the flow behavior around the different airfoils. The numerical results were validated by comparing the results with previouslyobtained experimental results by other researchers. The comparison showed good agreement. Various angles of attacks were considered. From the present study, the following conclusions may be set forth.

i. The airfoil SG6043 has the highest lift and lift to drag ratio.

ii. NACA0012 airfoil has the lowest lift and lift to drag ratio.

iii. The angle of attack has a direct impact on lift and drag.

iv. For the lift coefficient, the maximum value occurs at angle of attack $=15^{\circ}$.

v. For the drag coefficient, the minimum value occurs at angle of attack $=0^{\circ}$.

vi. For the lift to drag ratio, the maximum value occurs at angle of attack $=5^{\circ}$ for $\mathrm{SG6043}$ airfoil and $=10^{\circ}$ for NACA0012 and NACA2412.

vii. As the angle of attack increases the low pressure and weak region shifts gradually from the lower surface to the upper surface.

viii. The two airfoils NACA0012 and NACA2412 have symmetrical contours for the velocity and turbulent kinetic energy contours in contrast with the SG6943 airfoil.

ix. The wake region is stronger for the SG6043 profile than NACA2412 and NACA0012 profiles at same angle of attack.

$\mathrm{x}$. It is highly recommended to use SG6043 airfoil for wind turbine applications due to the high value of lift to drag ratio exhibited by this profile.

\section{References}

[1] Hossain, Altab, Ataur Rahman, A. K. M. P. Iqbal, M. Ariffin, and M. Mazian. "Drag analysis of an aircraft wing model with and without bird feather like winglet." International Journal of Aerospace and Mechanical Engineering 6, no. 1 (2012): 8-13.

[2] Mineck, Raymond E. Wind-tunnel investigation of aerodynamic efficiency of three planar elliptical wings with curvature of quarter-chord line. Vol. 3359. NASA, Scientific and Technical Information Program, 1993.

[3] Haque, M. Nazmul, Mohammad Ali, and Ismat Ara. "Experimental investigation on the performance of NACA 4412 aerofoil with curved leading edge planform." Procedia Engineering 105 (2015): 232-240.

https://doi.org/10.1016/i.proeng.2015.05.099

[4] Mahmud, Md Shamim. "Analysis of Effectiveness of an Airfoil with Bi-camber Surface." International journal of Engineering and Technology 3, no. 5 (2013): 569-577.

[5] Kandwal, S., and S. Singh. "Computational fluid dynamics study of fluid flow and aerodynamic forces on an airfoil." International Journal of Engineering and Technology 1, no. 7 (2012): 1-8.

[6] Pinkerton, Robert McLean. The variation with Reynolds number of pressure distribution over an airfoil section. US Government Printing Office, 1938.

[7] Sharma, Aman, Satish Guide Kumar, and Dwarika Nath Guide Ratha. "Evaluation of Flow Behavior around an Airfoil Body." PhD diss., 2012.

[8] Anderson, John D. "McGraw-Hill Series in Aeronautical and Aerospace Engineering." Fundamentals of Aerodynamics, by (2003): 54-56.

[9] Venkatesan, S. P., V. Praveen Kumar, M. Sunil Kumar, and Suraj Kumar. "Computational Analysis of Aerodynamic Characteristics of Dimple Airfoil NACA 2412 at Various Angles of Attack." idea 46 (2018): 10.

[10] Sogukpinar, Haci, and Ismail Bozkurt. "Calculation of Aerodynamic Performance Characteristics of Airplane Wing and Comparing with the Experimental Measurement." International Journal of Engineering Technologies 1, no. 2 (2015): 83-87. https://doi.org/10.19072/ijet.105718 
[11] Sogukpinar, Haci. "The effects of NACA 0012 airfoil modification on aerodynamic performance improvement and obtaining high lift coefficient and post-stall airfoil." In AIP conference proceedings, vol. 1935, no. 1, p. 020001. AIP Publishing LLC, 2018. https://doi.org/10.1063/1.5025955

[12] Sogukpinar, Haci, and Ismail Bozkurt. "Finding optimum airfoil shape to get maximum aerodynamic efficiency for a wind turbine." In AIP Conference Proceedings, vol. 1815, no. 1, p. 040007. AIP Publishing LLC, 2017. https://doi.org/10.1063/1.4976376

[13] Yemenici, Onur. "Experimental investigation of the flow field around naca0012 airfoil." International Journal of Sciences 2, (2013): 98-102.

[14] Rajakumar, S., and D. Ravindran. "Computational fluid dynamics of wind turbine blade at various angles of attack and low Reynolds number." International Journal of Engineering Science and Technology 2, no. 11 (2010): 64746484.

[15] Mehdi, Husain, Shwetanshu Gaurav, and Mudit Sharma. "Numerical investigation of fluid flow and aerodynamic performance on a 2D NACA-4412 airfoil." International Journal of Research in Engineering and Innovation 1, no. 1 (2016): 1-5.

[16] El-Sayed, Ahmed F. Aircraft propulsion and gas turbine engines. CRC press, 2008. https://doi.org/10.1201/9781420008777

[17] Menter, Florian R. "Two-equation eddy-viscosity turbulence models for engineering applications." AIAA journal 32 , no. 8 (1994): 1598-1605. https://doi.org/10.2514/3.12149

[18] Harris, Charles D. "Two-dimensional aerodynamic characteristics of the NACA 0012 airfoil in the Langley 8 foot transonic pressure tunnel." (1981). 